\title{
The Development of an On-Line Virtual Educational Support and Social Interface Link (VESSIL) System for Parents of Children Presenting with an Autistic Spectrum Disorder (ASD) Designed to Support Inclusive Education
}

\author{
Sian Benedict-Owen \\ Brunel University \\ United Kingdom
}

\begin{abstract}
Educating a child with an ASD dictates that where possible daily opportunities are created in order to teach and generalise skills across different environments. The extension of school-initiated interventions into the home is proven to lead to more positive outcomes for the child. However the effects of stress experienced by parents of a child with an ASD can impact enormously on their capacity to engage with their child's education. Research has indicated that high levels of parenting stress can counteract the effectiveness of teaching interventions. For the purpose of this study designed to test the prototype of a parent training and social networking system, the system was implemented at a school for children and young people presenting with moderate and severe learning difficulties where a significant proportion of students have a diagnosis of Autism. The aim of the system was to provide for parents engaged with the project a parent training platform and community focus constructed on social networking principles.
\end{abstract}

\section{Introduction}

Autistic Spectrum Disorders (ASDs) are a group of neuro developmental disorders which impact on aspects of an individual's neuro development and functioning competence. They affect areas of a person's processing in relation to social interaction, social communication and flexibility of thought. These are expressed in the International Statistical Classification of Health Related Problems 10th revision as:

'A group of disorders characterized by qualitative abnormalities in reciprocal social interactions and in patterns of communication, and by a restricted, stereotyped, repetitive repertoire of interests and activities. These qualitative abnormalities are a pervasive feature of the individual's functioning in all situations.'

This trio of commonalities when present within the regions of social interaction and communication and rigid, ritualistic behaviours is referred to as the 'triad of impairments' [27]. Autism is currently estimated to affect one in every one hundred children in the United Kingdom with four times as many boys diagnosed than girls [16].

The way in which an ASD affects the individual varies considerably from one person to another. Consequently parenting a child who presents on the spectrum is not the same for every parent. Experiences and needs will at times be different as well as sometimes in common. Dr Stephen Shore [25], Professor at Adelphi University, whose research focus is matching best practice to the needs of people with autism, states 'If you have met one person with autism - you have met one person with autism.'

Parental experiences can include a range of emotional and physical challenges including feelings of parenting inadequacy, social isolation, family breakdown and mental illness [9]. There can also be an expectation on parents, self-imposed or otherwise, for them to engage intensely with their child's programme of educational interventions, such as in Applied Behaviour Analysis where intervention can involve several hours of focused one on one engagement with their child each day.

Furthermore, when discussing parental engagement with educational interventions for children with an ASD, it is important to remember that the educational interventions alluded to can also include those of the wider 'life curriculum', for example life skills such as dressing, toileting and feeding. These types of interventions can be necessary for a child presenting with an ASD when they are of an age considerably more advanced than that of their neuro typical peers when they achieved these same skills.

Alongside ASD, an individual can have the existence of a co-morbidity with another condition such as Fragile X, Downs Syndrome and/or learning difficulties. A circumstance which can add an additional challenge to parents and impact on their coping mechanism and sense of emotional wellbeing. 


\section{Considering the Effect of ASDs on Parental Efficacy}

Whilst it is recognised that some parents may become imbued with a sense of energy at the prospect of supporting a child diagnosed with an ASD, having a child who presents with any kind of disability can prove stressful for family members, particularly parents [13].

Research has suggested that amongst parents of children with a disability the stress levels shown by parents of a child with an ASD are substantially higher than in the other groups of parents and that this stress could affect the child's success within an early intervention programme [3] [17] [20]. Divorce rates are reported to be higher amongst this group of parents [7]. Ambitious about Autism a national charity in the United Kingdom for children and young people with autism estimates the divorce rates of parents of children with autism as $60 \%$ higher than the average for other groups. One UK study found that one in three families of children diagnosed with ASD were headed by single parents [2]. Behaviour is also cited as a centrally problematic concern.

Other parental concerns adding to the pressures experienced by parents include: - worry about their child's ability to function independent of others

- ASD impacts on financial stability due to treatment costs and planning for a child's future when parents are no longer available to support them

- ASD impacts on employment status for parents when they have to miss significant amounts of time from work in order to attend appointments

- ASD impacts on a family's quality of life families are more likely to eat at home, not engage so much in community activities and spend more time on daily activities

- They might also receive little support from other extended family members

- worry about what quality of life their child will have as an adult and how they will cope when parents die

- health of their child

- often transportation issues

- lack of access to child minding

- language barrier

- difficult work schedules

- parental uncertainty as to what their role in the process is

- an absence of confidence when working in partnership with the different professionals involved in supporting their child.

The figure of carers of children with autism in full time work have been reported at $11 \%$ with $70 \%$ of carers saying that the lack of appropriate care facilities stops them working [1].

\section{Importance of Parental Engagement with Educational Interventions}

There have been a wealth of studies undertaken where the outcome has highlighted the effectiveness of implementing early teaching interventions for children with an ASD and the associated potential for more positive long term outcomes [12] [19] [21].

Karst, J.S et al. Jordan and Powell emphasised the importance of parental involvement in the learning and teaching programmes of children with ASDs describing it as 'imperative' in order to foster consistency between all environments for the children [8] [9]. Parents possess important information about their child which can contribute greatly to the reciprocity of the learning and teaching process [6]. Best practice suggests that parental involvement is needed both for more rapid advances and to ensure consistency of approach [15].

Whilst interventional approaches adopted can be seen to vary, those interventions identified by the National Research Council as comprehensive, all possess some degree of parental involvement within their model [15]. The most common parent participation model being that of parent as educator where parents are taught intervention techniques which enable them to continue interventions in the absence of professional input. As educators, parents are in a strong position to address issues concerned with generalising of their children's learning over a range of natural settings and situations including those within the home and the wider community. In addition Rao and Beidel put forward the view that as well as treating the child presenting with an ASD, parents may also need to learn effective strategies for coping with their own distress [18].

\section{School - Roles, Responsibilities and Inclusive Education}

Schools and educational authorities in the United Kingdom are obliged by law to develop cultures, policies and practices which work towards achieving an inclusive educational environment. Wilkinson and Twist within their work on UK Policy and Practice in relation to the education of children and young people with autism emphasise the legal expectation of schools to adopt attitudes and processes that make sure that all pupils have equal access to the curriculum [24] [26].

Additionally, Wilkinson and Twist concluded within their work concerning educational provision and autism that [24]:

'despite best endeavours, it appears that efforts towards achieving mainstream educational inclusion 
of pupils identified with ASD still have some way to go. In practice, higher school exclusion rates and greater gaps in attainment for ASD pupils compared to their typically developing peers demonstrate a need for improvements in educational provision for these pupils'

The above findings suggest that currently there exists a discernable gap within schools' inclusive provision for this cohort of children. As discussed elsewhere, the effective education of young people presenting with an ASD requires an informed, consistent and 24 hour curriculum approach. Possibly the lack of access to such a consistent and focused curriculum by this cohort of children, could in part, be responsible for the issues outlined above. The provision of an intervention by schools which facilitated parents to become empowered, confident and informed educators of their child, as part of the school's inclusive approach could prove part of the answer to those concerns raised by Wilkinson and Twist [26].

\section{Research Plan}

This research was planned as the first phase in a study seeking to design a system which could be implemented on-line and used by parents as an educational knowledgebase and social interaction system. Anticipated outcomes included identifying whether and to what extent a targeted virtual educational and community network system could present as a meaningful tool for parent training, information sharing and as an emotional well-being conduit for parents of a child with an ASD.

Put simply the intention of this study was to develop and test a system's design which was proposed as a possible educational and social networking intervention for parents of children presenting with ASD. Its design based on the proposition that better overall outcomes will be achieved by children with ASDs when their parents are informed, empowered, engaged and supported. A further aim at this point of the research was that implementation of, and engagement with, the prototype would provide a basis for additional information gathering from parents and educators to identify additional system requirements which could be added into the system model. Implementing and administering the modified model would then underpin the foundations of phase two of an extended project.

\section{Learning Theory}

In 2005 Siemens amongst others questioned the appropriateness of current learning theories within a changing technological landscape [5]. He argued that the rapid growth and complexity of information available on the internet, the ability for individuals to interact on global networks, and the ability to combine different information flows suggested a need for a new learning theory. A new learning paradigm was needed in order to capture the new patterns of learning dynamics. Siemens suggested that the starting point for learning transpires when knowledge is triggered through the process of a learner linking with and submitting information into a learning community.

In the connectivist model, a learning community is termed a 'node'. Each 'node' is always part of a larger network. 'Nodes' are the result of connection points existing on a network. Each network consists of two or more 'nodes' which are connected for the purpose of sharing resources. Learners connect to a network to share and find new knowledge. The connectivist approach recognises that by increasing subject competence and the discovery by the learner of additional facts within a subject area, current knowledge held may change. A learner's existing knowledge will be extended and or modified on the basis of newly gained knowledge and subject competence. This new knowledge is then shared within the network. This modified knowledge may then itself be altered and shared as a result of further new knowledge received via the network. The skills regarded as imperative to learning within the connectivist stance are the ability to find current information and the ability to filter secondary and irrelevant information. Siemens argued 'the capacity to know is more critical than what is actually known' [24].This connectivist approach presents a clear and germane paradigm upon which the aspirations of the learning dynamic framed within this research are based.

\section{Methodology}

The project was piloted in a geographically defined area. All of the children whose parents took part in the study attend the same local authority special school in an outer London borough. The school offers specialist provision for children and young people with moderate and severe learning difficulties. The school also has its own autism provision.

Initial contact was made by the researcher with the head teacher of the school outlining the research intention and a meeting date was arranged. At the meeting a discussion was conducted between the head teacher, the school's Co-ordinator for the management of the Social Communication, Emotional Regulation and Transactional Support (SCERTS) programme running in school and the researcher. It was established between the head teacher and the SCERTS co-ordinator that the coordinator had capacity to input to the 'VESSIL' system on a daily basis with up to five parents. 


\section{Instruments of Measure}

A 'parental/ carer anxiety assessment sheet' was developed by the author. This was designed for the purpose of identifying levels of parental anxiety about a range of issues such as their child's social development, ability to communicate, self-injurious behaviours and diet before the intervention was in place. This assessment was completed by parents at the beginning of the study. The 'parental/carer anxiety assessment sheet' could then be completed again at a later date and the data compared in order to identify if there had been a reduction in levels of parental anxiety during the period that parents accessed the intervention.

Semi-structured interviews were also employed as a method of identifying parent's perceptions as to their parental concerns regarding their child and their education, their relationship with their child's teacher and the degree which they, as parents, engaged with school.

\section{Study Population}

The sample was selected using a purposive method and was comprised of parents of children presenting with an autistic spectrum disorder. The SCERTS Coordinator identified five children that she was currently working with. Details about the research and an invitation to participate was sent home to parents in the form of a pamphlet.

All of the parents approached agreed to take part in the research. Three actively did so. An initial forum meeting was held at the school with those parents who expressed an interest in taking part. At the meeting expectations of user etiquette were established. Parents would 'touch-base' on-line at least three times a week, even if just to say 'hello'. This proviso was to encourage parents to become familiar with accessing and using the intervention through frequency of use.

\section{Consent}

A letter in the case of school-side and pamphlet in the case of parents, was sent prior to any initial engagement in keeping with good ethical practice. The overall aims of the project in general terms was explained and recipients were asked whether they would be agreeable to participate.

\section{System Considerations and Functionality}

A set of systems considerations was produced. Considerations included the identification of an existing social networking site which would allow for the prototype of the on-line Virtual Educational
Support and Social Interface Link (VESSIL) System to be created using the host network's own functionality. The reasons for sitting the 'VESSIL' system within an existing social networking site at this pilot stage included deliberations such as the cost of developing and maintaining a pilot site, the ease of accessibility to the site by parents and how easily security levels could be implemented. Another attribute of operational functionality at this initial stage was to have a facilitator from the school's own teaching staff as well as the researcher. This offered a twofold benefit. Parents would have the familiarity of a facilitator they already knew and also one who could respond more immediately to requests directed

'school-side'.

It was essential that the 'VESSIL' system would be able to provide secure levels of protection of any information shared.

The system requirements identified were felt most closely to match Google+ capabilities. Thus Google+ was selected as the host network for the pilot. Each member of the pilot sample needed to either have a pre-existing Google+ account or to set one up for the purpose of the study.

Once a Google+ account is in place, permanent links can be created with other account holders by linking email addresses. In Google+ terms this process is regarded as the creation of a 'circle'. For the purpose of the pilot, 'circle' permutations could consist of a variation of different variables such as the two facilitators, the researcher and the SCERTS Coordinator, the researcher-the SCERTS Coordinator/parent/s, parents together.

\begin{tabular}{|c|c|}
\hline Facilitator 1 & Facilitator 1 \\
Facilitator 2 & $\begin{array}{c}\text { Facilitator 2 } \\
\text { Parent }\end{array}$ \\
\hline Facilitator 1 & Parent 1 \\
Facilitator 2 & Parent 2 \\
Parent/Parent & Parent 3 \\
\hline
\end{tabular}

Figure 1. Examples of Possible Group Permutations

A mirror image of each 'circle' needed to be created by all members of a discreet group. Initial 'circles' were set up by participants under the guidance of the researcher. Every 'circle was codified using a unique name. In the initial stage, each parent could access a 'circle' consisting of themselves, the researcher and the school side facilitator. The system design allowed for other 'circles' to be created based on different member permutations. If a post was addressed to a particular named 'circle', the contents of the post would only be received by those who were designated members of that discreet 'circle' by the initiator of the post sent. The flexibility of the 'circle' design allowed that 'circle' permutations could be modified on a post by post basis if this was required. Posts sent to a 
'circle' gave members of that 'circle' the opportunity to share comments discreetly across the group. Posts sent within a 'circle' allowed all members of the 'circle' to also see any additional comments made within the 'circle' which were linked to the post and also an opportunity to share further comments, photos etc. within the group.

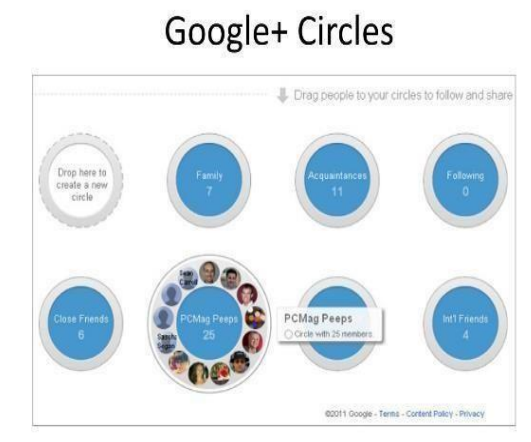

Figure 2. Google Circles

Whilst information sharing by account holders of Google+ at a public level is possible, the use of the Google+ 'circles' capability ensured that information sharing could be controlled and shared on a predetermined level. All individual members could choose who would be a member of a particular 'circle' and control the flow of any information they themselves shared.

Project issues were also influenced by the findings of Losh et al., namely that sub-threshold characteristics of autism are often seen in the parents of children presenting with an ASD, i.e. they are part of the broader autism phenotype (BAP) so that they too could present with their own difficulties around indicators of ASD [11].

A possible parental BAP connection raised its own design concerns as parents presenting with the broader autism phenotype might themselves gain greater benefit from an end user interface which was markedly logical in its operational structure and supported by very clear and uncluttered visual graphics. For the 'VESSIL' system to be perceived by parents as a helpful tool it was important that the system was easy to access and navigate.

Certain strictures of use and etiquette protocols also needed to be identified and agreed to ensure that the system was used for its design purpose and any misuse such as being used as a tool to voice dissatisfaction by parents about teaching staff or the school facilities could be avoided.

Table 1. Structures of Use and Etiquette Protocols

\begin{tabular}{|l|l|}
\hline Facilitators & Parents/carers \\
\hline $\begin{array}{l}\text { That the community } \\
\text { should be a place which }\end{array}$ & $\begin{array}{l}\text { The group was a place } \\
\text { where they could discuss }\end{array}$ \\
\hline
\end{tabular}

\begin{tabular}{|l|l|}
\hline $\begin{array}{l}\text { offered a supportive and } \\
\text { confidential environment }\end{array}$ & $\begin{array}{l}\text { and share their concerns } \\
\text { about their child in } \\
\text { confidence with } \\
\text { professional teachers }\end{array}$ \\
\hline $\begin{array}{l}\text { Comments made within } \\
\text { the group should be } \\
\text { confined to issues } \\
\text { relating to the specific } \\
\text { child and their education }\end{array}$ & $\begin{array}{l}\text { Comments made within } \\
\text { the group should be } \\
\text { those concerning their } \\
\text { own child and their } \\
\text { education and not } \\
\text { addressing wider school } \\
\text { issues, i.e. This was not } \\
\text { a forum for recording } \\
\text { dissatisfaction about } \\
\text { school related issues }\end{array}$ \\
\hline $\begin{array}{l}\text { Facilitators should avoid } \\
\text { offering advice which } \\
\text { contradicted or } \\
\text { conflicted with that of } \\
\text { the child's class teacher }\end{array}$ & $\begin{array}{l}\text { That with parents' } \\
\text { permission 'facilitators' } \\
\text { might at times consult } \\
\text { with members of the } \\
\text { schools teaching team }\end{array}$ \\
\hline $\begin{array}{l}\text { Group members should } \\
\text { act with integrity } \\
\text { towards each other and } \\
\text { respect each other's } \\
\text { privacy }\end{array}$ & $\begin{array}{l}\text { That group members } \\
\text { would act with integrity } \\
\text { towards the other } \\
\text { members of the group } \\
\text { and respect their privacy }\end{array}$ \\
\hline $\begin{array}{l}\text { Comments made within } \\
\text { the group should not } \\
\text { address issues relating to } \\
\text { wider school concerns }\end{array}$ & \\
\hline
\end{tabular}

Exchanges could be expected during week days during term time in recognition that the school side facilitator was signed to a contract of employment and should not be expected to commit to engage in the study outside of contracted work hours.

\section{The On-Line Virtual Education Support and Social Interface Link (VESSIL) System - TouchBase121}

The system was staged to parents as a packaged product with a unique product name 'Touchbase 121 ' and its own logo. This was done in order to give the system an element of prestige aimed to heighten parents confidence. Rather than perceiving the system as being a 'work in progress' it should be viewed as an already capable product.

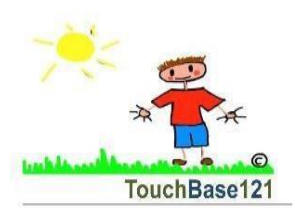

Figure 3. TouchBase121 - Logo 
This was important as in order for parents to feel supported by the system they needed to have the confidence that it would be effective. It was hoped that the system would be seen to grow organically with new 'circles' being created and that relationships between members of 'circles' would develop and perhaps deepen.

The 'VESSIL' system was designed with the purpose of information provision and the amelioration of parental stress and anxiety through the provision of both parent training and social links between groups: educators and parents and parents with each other.

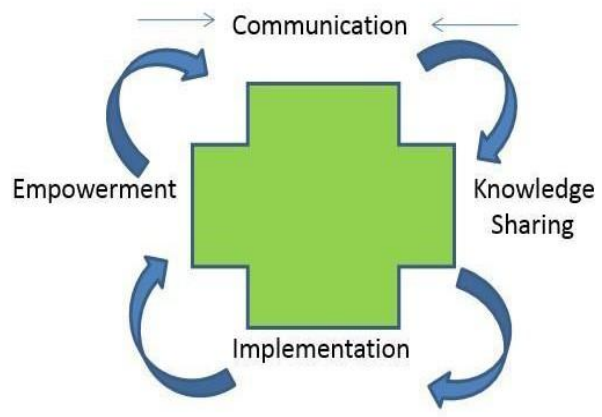

Figure 4. Proposed System Dynamic - Cycle of Empowerment

Communication flows were designed to allow for multi way interactions. By the different types of knowledge sharing which could be achieved through the medium of the system, e.g. parent training (one of the centrally proposed functions of the communication flow) it was anticipated that parents should feel more confident at implementing those intervention strategies put in place by educators for the purpose of supporting their child's education. This confidence in turn could lead to increased perceptions of parental efficacy and well-being leading to increased feelings of parental empowerment. Ideally parental stress levels should then be favourably impacted on which in turn could allow for parents to feel more able to engage with their child's educational interventions. And so the cycle should continue.

\section{The Study}

An initial meeting was held at the school. Three parents and the SCERTS co-ordinator attended. Feedback from the group included that parents were looking forward as much to the social aspect of the project as well as of any educational benefits that might be gained. At the meeting parents were asked to complete the parent/carer anxiety assessment sheet'. Parents were shown how to sign up to Google+ and create a 'circle'. Both the researcher and the school side 'facilitator' posted onto the system each morning. Posts varied from those with
ASD specific educational information to pictures accompanied by texts containing statements aimed at encouraging feelings of self-empowerment The community used the network for a variety of different reasons, some relating to questions of behaviour 'I have to take $J$. to a wedding next Friday and I am a bit concerned that he might freak out', and communication strategies, 'How do you know what's wrong with your child... everyone says to take him to the doctor but I don't know what to tell him'. Other enquiries were specifically aimed at other parents such as asking what they would be doing over the holiday periods with their child, what were their experience of accessing respite services: 'Question: Do parents here get respite for your children? If so, how did you go about getting it?' (Parent 3).

Photographs and web links were shared between parents about their children and their experiences: 'This short clip gives me insight into (name)'s world.

I watch him closely when we go out for our walks and I think this is how things are for him...' (Parent 1)

The pilot was managed over a half term school period of six weeks by the researcher. For the purposes of researching the prototype system, the researcher felt that by that time, the aims of the pilot system for research purposes had been met. The second forum meeting was planned as a final feedback session. Both the parents and the school side 'facilitator' expressed that they were reluctant to close down the system at this point and requested that it continue as an extension of the current communication systems in place between home and school.

For the next period of a term the researcher acted as an observer of the functioning system.

\section{Findings}

It was clear from feedback given by parents both in written form and through forum discussions that with regards seeking educational advice, parents were very happy with the current arrangements. As their children attended a school which itself delivered specialised educational provision they professed themselves satisfied with the available levels of educational support. They believed teachers at the school had both the right levels of specialist expertise in order to support the needs of their child and also the ability and willingness to meet the parents own information needs regarding any educational interventions so allowing them, as parents, to proficiently support their own child's educational interventions at home. The feature of the study outcomes that appeared of particular value to this set of parents was the social opportunities that the system offered parents, allowing them to engage with each other in a way that had not previously been 
possible. Within a period of weeks of the system being in place members of the system began communicating with each other during weekend and later the holiday periods.

'...do you think that other parents would benefit from using this? ....it's nice to chat and share thoughts with each other and I thought some other parents might like/need this too?' (Parent 3) Parents summarised their experiences of using Touchbase 121, experiences included:

'I have found 'TouchBase' a fantastic tool for making contact with school and other parents. I have found the information I have got here very useful. Even tho I am a prolific user of the home school book, I think 'TouchBase' fills the gap that the book alone does not...' (Parent 1) 'TouchBase 121 has given me more confidence in dealing with (name). I now have much needed understanding that other people experience the same issues as (name). It's the perfect platform to go and ask questions for any issues or concerns that I have. With this, I don't feel so alone! I would really like to thank you for inviting me to take part in this'. (Parent 2) '...TouchBase has helped voice immediate concerns and know someone will answer as soon as they can. I love that it has been kept and that we are now able to interact with other parents as well as professionals.' (Parent 3) The positive tone of these excerpts and the comments made clearly demonstrate that there is a place for this type of parental intervention.

\section{Conclusion}

In England there are reported to be $71 \%$ of children with autism being educated in mainstream schools with 54\% of teachers in England feeling that they have inadequate training to meet their needs [4] [14].

The above statistics combined with other findings cited in this article suggest that a modified parent training/social network system within a mainstream educational environment based on the principles of the current 'VESSIL' system could prove a useful intervention model for schools to adopt. A key objective being that parental access to the 'VESSIL' system in a mainstream school environment will lead a parent to feel that in relation to their child, and their own educational and social requirements as a parent, they are adequately supported and that this in turn will increase a parent's sense of parental efficacy, emotional well-being and reduce any feelings of anxiety and stress. Furthermore an increased sense of well-being should impact positively on parental capacity and willingness to engage more fully with their child's educational interventions thereby leading to more positive long term outcomes for the child.

By educating and empowering parents, effective educational partnerships could be developed across home/school environments which in turn could lead to more effective educational support mechanisms being put into place for children and young people presenting with ASD thereby perhaps lessening those causal incidents which ultimately lead to poor attainment and/or exclusion.

The modified 'VESSIL' system is intended to be pioneered in an outer London borough locality and operated on a 'cluster' principle whereby a group of neighbouring schools are linked into the same system. The system will be independent of any one school but will act as a central point, supporting the parents of children with an ASD who attend one of a group of mainstream primary schools within a denoted area.

\section{References}

[1] Broach, S., Camgoz, S., Heather, C., Owen, G., Prior, A. (2003) Autism Rights in Reality . National Autistic Society

[2] Bromley, J., Hare, D, Davison, K. and Emerson, E. (2004). Mothers supporting children with autistic spectrum disorders: social support, mental health status and satisfaction with services. Autism, 8(4), 409-423

[3] Carlson-Green, B., Morris, R.D., Krawiecki, N. (1995). Family and Illness Predictors of Outcome in Pediatric Brain Tumours. Journal of Pediatric Psychology, vol. 20, no. 6, pp. 769-784,

[4] Department for Education (2012). Special Educational Needs in England

[5] Downes. S. (2005, December 22). An introduction to connective knowledge. Stephen's Web. http://www.downes.ca/cgi-bin/pagecgi?post=33034

[6] Govt. Publications Dublin (2006). An Evaluation of Educational Provision for Children with Autistic Spectrum Disorders). Dept. Education and Science 3.4.4.

[7] Hartley, S. L., Barker, E. T., Seltzer, M. M., Greenberg, J., Bolt, D., Floyd, F., \& Orsmond, G. (2010). The relative risk and timing of divorce in families of children with an autism spectrum disorder. Journal of Family Psychology, 24, 449-457.

[8] Jordan, R., Powell, S.(1995). Understanding and Teaching Children with Autism. England: Wiley and Sons

[9] Karst J.S et al. 2012. 'Parent and Family Impact of Autism Spectrum Disorders: A Review and Proposed Model for Intervention Evaluation'. Clinical Child and family Psychology Review, 15 - 247-277

[10] Levy, S., Kim, A., \& Olive, M.L. (2006). Interventions for young children with autism: A synthesis of the literature. Focus on Autism and Other Developmental Disabilities, 21(1), 55-62 
[11] Losh, M., Childress, D., Lam, K., \& Piven, J. (2008). 'Defining key features of the broad autism phenotype: a comparison across parents of multiple and single-incidence autism families'. American Journal of Medical Genetics Part B: Neuropsychiatric Genetics, 147B (4), 424-433

[12] Lovaas, O.L. (1987). Behavioural Treatment and Normal Educational Functioning in young Autistic Children. Journal of Consulting and Clinical Psychology, $55,3-9$

[13] National Autism Centre. (2012). A Parent's Guide to Evidence-Based Practice and Autism. Massachusetts, National Autism Center http://article.wn.com/view/2012/06/09/A_Parents_Guide_t o_EvidenceBased_Practice_and_Autism/

[14] National Foundation for Educational Research (2011). Teacher Training on Autistic Spectrum Disorders. Teacher Voice Omnibus February 2011 Survey

[15] National Research Council. (2001) Educating Children with Autism. Washington, DC: national Academy Press

[16] Office of National Statistics (2005). Mental Health of Children and Young People in Great Britain. London: Palgrave McMillan

[17] Osborne, L.A., McHugh, L., Saunders, J. and Reed, P. (2008). Parenting Stress Reduces the Effectiveness of Early Teaching Interventions for Autistic Spectrum Disorders. Journal Autism and Developmental Disorders, 38:1092-1103

[18] Rao, P.A. \& Beidel, D.C. (2009). 'The Impact of Children with high-functioning autism on parental stress, sibling adjustment, and family functioning'. Behaviour Modification, 33, 437-451

[19] Reed, P., Osbourne, L.A. and Corness, M. (2007). Relative effectiveness of different home based behavioural approaches to early teaching intervention. Journal of Autism ND Developmental Disorders, 37, 1815 - 1821

[20] Robbins, F.R., Dunlap, G., and Plienis, A.J. (1991), Family Characteristics, Family Training and the progress of young children with autism. Journal of Early Identification, 15, 173-184

[21] Sallows, G.O. and Graupner, T. D. (2005). Intensive Behavioural Treatment for Children with Autism: four year outcome and predictors. American Journal on Mental Retardation, 110, 417-438

[22] Shore, S, http://www.autismasperger.net/intro.htm

[23] Schultz, T.R., Schmidt, C.T., \& Stichter, J.P. (2011). A Review of Parent Education Programs for Parents of Children with Autism Spectrum Disorders. Focus on Autism and Other Developmental Disabilities, 26(2) 96104

[24] Siemens. G. (2005, August 10) Conectivism: Learning as Network Creation. E-learning space.org.website.

http://www.elearnspace.org/Articles/networks.htm
[25] Siemens, G. (2008) About: Description of Connectivism. Connectivism: A learning theory for today's learner, website. http://www.connectivism.ca/about.html

[26] Wilkinson, K. and Twist, L. Autism and Educational assessment: UK Policies and Practice: Slough:NFER.

[27] Wing, L. and Gould, J (1979). 'Severe Impairments of Social Interaction and Associated Abnormalities in Children'. Journal of Autism and Developmental Disorders, 9, pp 111-29 